\title{
Physical views on ParABS-mediated DNA
}

\section{segregation}

B. Guilhas ${ }^{1}$, A. Le Gall ${ }^{1}$, M. Nollmann ${ }^{1 \#}$

1 Centre de Biochimie Structurale, CNRS UMR 5048, INSERM U1054, Université de Montpellier, 60 rue de Navacelles, 34090, Montpellier, France

${ }^{*}$ Corresponding author

\begin{abstract}
In this chapter, we will focus on ParABS: an apparently simple, three-component system, required for the segregation of bacterial chromosomes and plasmids. We will specifically describe how biophysical measurements combined with physical modeling advanced our understanding of the mechanism of ParABS-mediated complex assembly, segregation and positioning.
\end{abstract}

\footnotetext{
Abbreviations

DNA: deoxyribonucleic acid

GTP: guanosine triphosphate ATP: adenosine triphosphate NTP: nucleoside triphosphate CBP: centromere binding protein Ori: replication origin region Ter: replication termination region nsDNA: non-specific DNA $\mathrm{Kb}$ : kilobase
} 


\subsection{Introduction}

Faithful inheritance of genetic information is critical for cell viability. This process specifically requires the efficient and robust segregation and organization of DNA molecules within the sub-cellular space of the cell. To this end, most bacteria make use of active partitioning systems. These systems are typically are composed of three factors: a centromere DNA sequence, a centromere binding protein (CBP), and a NTPase. The specific DNA sequence recruits an assemblage of the CBP that self-assembles into a macromolecular complex called the partition complex. This complex is segregated by the NTPase motor protein. These partitioning systems have been classified into three groups according to their NTPase (J.-Y. Bouet \& Funnell, 2019; Brooks \& Hwang, 2017): Type I systems are based on Walker-type ATPases, Type II code for actin-like ATPases, and Type III systems use tubulin-like GTPases.

ParABS systems are members of the Type la partition system (Ebersbach \& Gerdes, 2005), a subdivision of the type I class that partly emerges from specific properties of the centromere binding protein. Binding of the CBPs (typically called ParB) to centromeric sequences (called parS) lead to the robust formation of partition complexes (also called ParBS complexes, Figure 1A). Once DNA is replicated, parS-containing DNA molecules are split, segregated, and positioned at specific sub-cellular locations. These processes typically require the action of ParA, the motor protein (Ah-Seng, Rech, Lane, \& Bouet, 2013; Debaugny et al., 2018) (Figure 1B). ParABS systems are conserved in more than $60 \%$ of sequenced bacterial species, and are the most widespread in bacteria (Gerdes, Møller-Jensen, \& Bugge Jensen, 2000; Livny, Yamaichi, \& Waldor, 2007). While other partition systems are exclusively dedicated to plasmid partitioning, ParABS systems segregate both plasmids and bacterial chromosomes (Badrinarayanan, Le, \& Laub, 2015; Baxter \& Funnell, 2014; J.-Y. Bouet \& Funnell, 2019). 

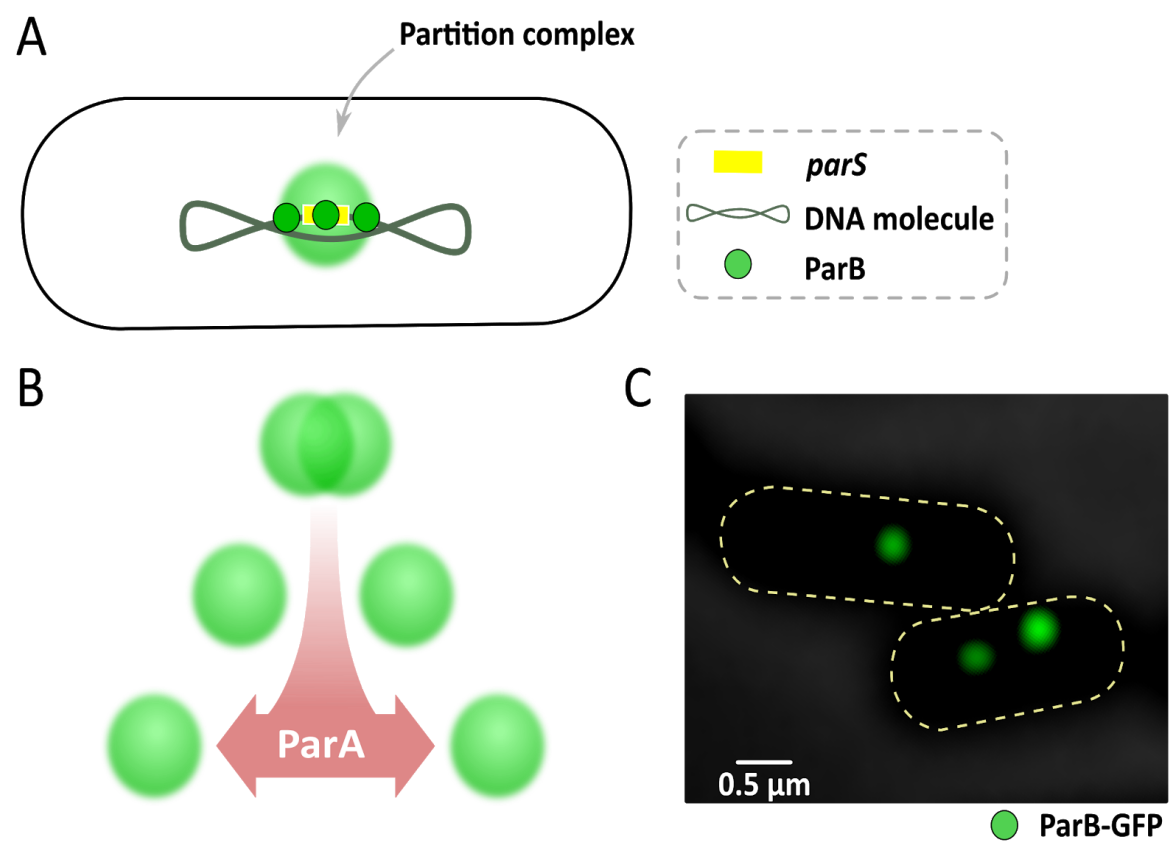

Figure 1. ParABS-mediated segregation of DNA molecules. (A) parS sequences (yellow) recruit several hundreds of ParB proteins (green sphere) to form the partition complex (green gradient). (B) Segregation of partition complexes by the ParA ATPase (red arrow) leads to the separation of DNA molecules harbouring parS. (C) Partition complexes can be imaged by visualizing ParB-GFP by fluorescence microscopy (image shows ParB-GFP from F-plasmid in E.coli). Partition complexes appear in green, and cell contours are represented with yellow, dashed curves.

\section{Chromosomal ParABS systems}

Bacteria are among the smallest forms of cellular life. To face the challenge of packaging their relatively large genome without compromising cellular processes, they display a complex internal organization (Figure 2). This ensures that, upon cell division, cellular checkpoints may be applied in space and time to ensure that low copy number components are evenly distributed among the daughter cells. Unlike eukaryotes, bacteria generally have only one chromosome that replicates concomitantly with cell growth. Bacterial chromosomes generally fold into two main configurations: longitudinal or transverse (Badrinarayanan et al., 2015; Baxter \& Funnell, 2014; J.-Y. Bouet \& Funnell, 2019)). In the longitudinal configuration, the origin (Ori) and terminus (Ter) of replication are located at opposite ends of the cell with left and right chromosomal arms between the two, arranged side by side. In the transverse configuration Ori and Ter are located at the middle of the cell with the left and right arms arranged in separate cell halves. In either case, the origin and terminus of replication are located at specific locations in the cellular 
space that must be restored during replication and maintained once replication is completed. Chromosomal ParABS systems serve both for the segregation and positioning of the duplicated origin proximal region of the chromosome and were recently identified as a hub for the loading of whole-chromosome organisers, namely the SMC-ScpAB complexes.

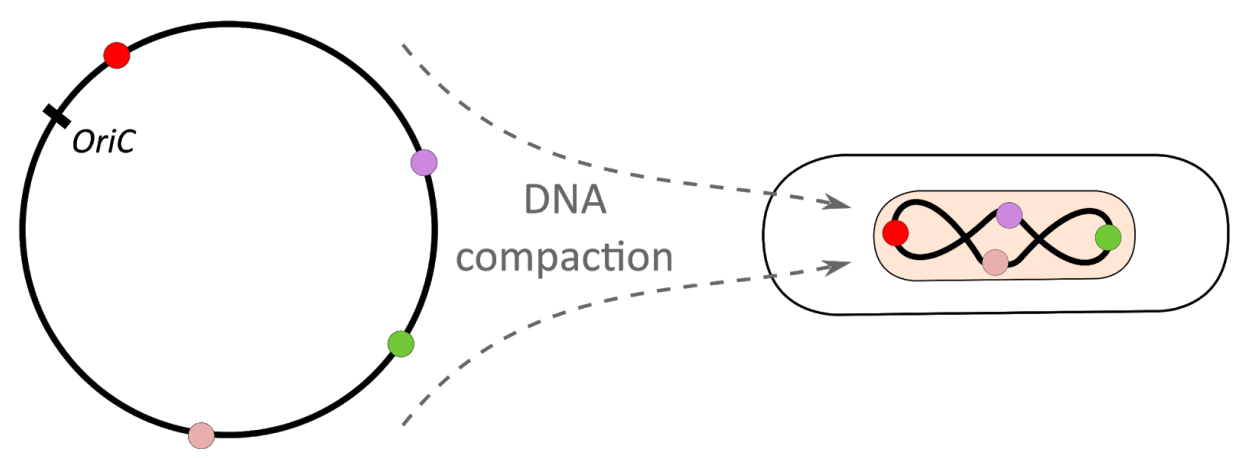

Figure 2. The topologically closed bacterial chromosome (circle in left panel) is compacted into the sub-cellular space following a well-defined choreography whereby each genomic region occupies a specific sub-cellular location. This positioning is controlled by different factors, including the ParABS system.

Most chromosomal parS sequences are located in the immediate vicinity of Ori (J.-Y. Bouet \& Funnell, 2019; Livny et al., 2007). When tagged with a fluorescent reporter, ParB forms, in vivo, bright fluorescent foci whose dimensions are diffraction limited (Figure 1). These ParB foci are referred to as partition complexes. The positioning and segregation pattern of partition complexes reflect the positioning of origin-proximal regions containing parS sequences, but vary among bacterial species. In Vibrio cholerae (Yamaichi, Fogel, \& Waldor, 2007), Caulobacter crescentus (Mohl \& Gober, 1997; Toro, Hong, McAdams, \& Shapiro, 2008) or sporulating Bacillus subtilis (Lin, Levin, \& Grossman, 1997; Webb et al., 1997), this region is anchored at the cell pole while the Ter is either anchored at the opposite side of the cell (notably in Caulobacter) or positioned at the cell center (e.g. B. subtilis, V. cholerae or Pseudomonas aeruginosa). Polar anchoring is mediated through direct interactions between ParB and polar proteins such as PopZ in C. crescentus (Bowman et al., 2008; Ebersbach et al., 2008) and HubP in V. cholerae (Yamaichi et al., 2012). In sporulating $B$. subtilis, ParABS-mediated segregation facilitates the RacA/DivIVA-dependent polar anchoring (Ben-Yehuda, Rudner, \& Losick, 2003; X. Wang \& Rudner, 2014). In Caulobacter, the ParABS system is essential for origin segregation and for the establishment of proper chromosome choreography. After replication and splitting of Ori regions, one of 
the two replicated origins migrates to the opposite pole, crossing the cell and the nucleoid from one pole to the other (Figure 3A) (Ptacin et al., 2014; Shebelut, Guberman, van Teeffelen, Yakhnina, \& Gitai, 2010; X. Wang \& Rudner, 2014). ParA is known to play a key role in this segregation process. In this system, the disruption of the motor protein ParA prevents the directed movement of Ori and impedes it from reaching the opposite pole (Shebelut et al., 2010). Using conventional fluorescence microscopy, ParA was shown to form a wave over the nucleoid that oscillates between cell poles, however the mechanism underlying this oscillatory behavior of ParA remains unclear. In vegetative B. subtilis, a similar process takes place, where replicated origins are segregated bidirectionally from midcell to $1 / 4$ and $3 / 4$ of the cell main axis (Lee, Lin, Moriya, \& Grossman, 2003; X. Wang, Montero Llopis, \& Rudner, 2014) (Figure 3B). The ParABS system plays important roles in this micrometric-scale translocation event, however it is not essential for cell viability. Lack of ParA leads to three defects in Ori segregation, namely an altered resolution characterized by a longer cohesion time between the two newly replicated origins (Lee, Lin, Moriya, \& Grossman, 2003), a loss in segregation featured by the abolishment of bi-directionnal movement, and a lack of robust positioning characterized by frequent backward movements of Ori towards midcell (X. Wang, Montero Llopis, \& Rudner, 2014).

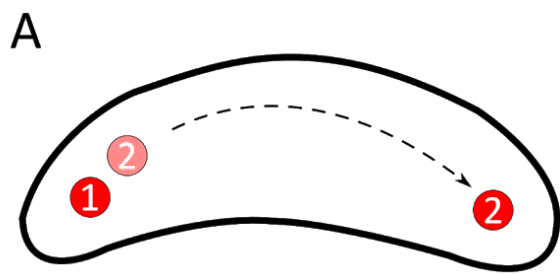

Caulobacter crescentus
B

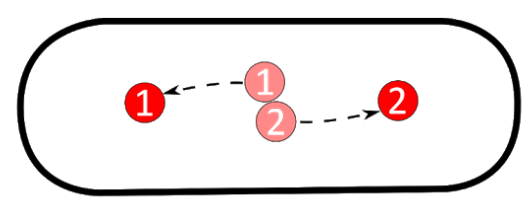

Bacilus subtilis
(1) Copy $\mathrm{n} 1$ of Ori

(2) Copy $\mathrm{n} 2$ of Ori

Figure 3. Models for the segregation of newly replicated origins in $C$. crescentus and $B$. subtilis. (A) In C. crescentus, the partition system is essential for the Ori to cross the cell. (B) In $B$. subtilis, the ParABS systems is required for proper resolution and segregation of replicated origins and is key to ensure the stable sub-cellular positioning of segregated origins.

Bacterial species harbouring ParABS systems typically rely on a second factor to properly segregate and localize chromosomes: the SMC-ScpAB complex (Gruber, 2018). This complex consists of two structural maintenance of chromosomes (SMC) subunits and two 
kleisin homologues (ScpA and $\mathrm{ScpB}$ ). SMC-ScpAB is recruited to Ori-proximal regions by specific interactions with ParB bound to parS (Figure 4A) (Gruber \& Errington, 2009; Miermans \& Broedersz, 2018; Sullivan, Marquis, \& Rudner, 2009; X. Wang, Brandão, Le, Laub, \& Rudner, 2017). In B. subtilis, SMC-ScpAB is required for the proper resolution of freshly replicated sister origins (Gruber et al., 2014; Lee et al., 2003; Marbouty et al., 2015; X. Wang, Tang, Riley, \& Rudner, 2014). Recently, the use of chromosome conformation capture technologies ( $3 \mathrm{C}$-seq) has revealed that SMC-ScPAB bridge long-range interactions between left and right replichores that extend from Ori to Ter (Figure 4A) (Marbouty et al., 2015; X. Wang et al., 2015). ParB, parS and SMC-ScpAB are all essential for bridging interarm interactions and participate in the proper establishment of the longitudinal organization of bacterial chromosomes (Marbouty et al., 2015; X. Wang et al., 2015). How the loading of SMC-ScpAB to Ori-proximal parS sequences leads to the global organization of the genome is still unclear. Current models point towards an active translocation of SMC-ScpAB along the chromosome by a mechanism with parallels with the DNA loop extrusion model (Alipour and Marko 2012). In addition to organizing the global folding of the genome, SMC-ScpAB plays a role in the spatial organization of the Ori-proximal region by bridging together distant parS sequences (Figure 4C) (Marbouty et al., 2015). Super-resolution microscopy showed that this spatial organization changes with the cell cycle and with the sub-cellular positioning of origins, suggesting that the $3 \mathrm{D}$ folding of the origin region could play a role in the regulation of replication initiation (Marbouty et al., 2015) (Figure 4B). Thus, in B. subtilis, both the ParABS system (X. Wang \& Rudner, 2014) and the SMC-ScpAB complex act in concert to achieve chromosome segregation and robust chromosome choreography (Marbouty et al., 2015; Miermans \& Broedersz, 2018; X. Wang et al., 2017, 2015). 


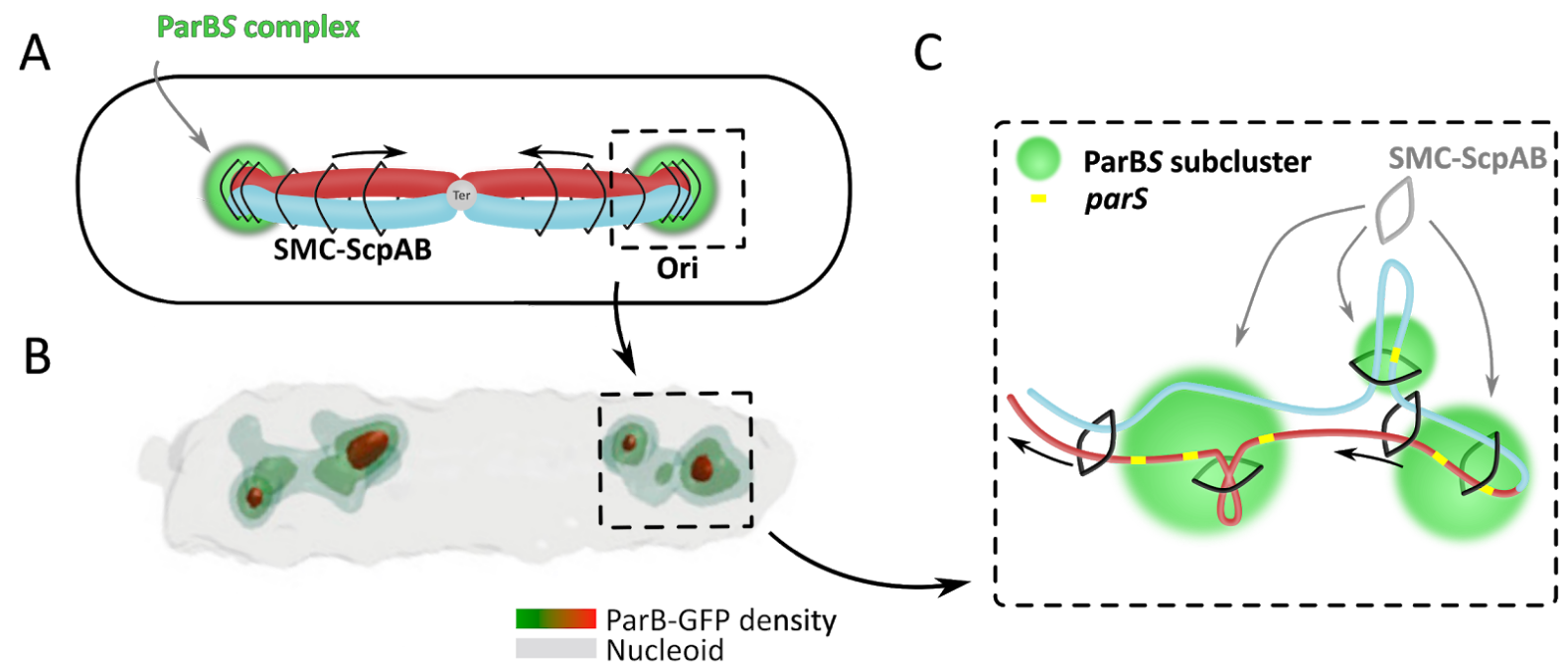

Figure 4. Compaction of $B$. subtilis chromosome mediated by ParBS and SMC-ScpAB complexes. (A) Condensin complexes (black arcs) are recruited by the ParBS partition complex (green) to Ori regions. They then slide or translocate towards the terminus of replication, and pair the two replication arms (blue and red) together. (B) 3D-structured Illumination Microscopy image of ParB-GFP in B. subtilis. (C) Schematic representation of the folding of the origin-proximal region. ParBS subclusters (green) are nucleated by parS sites (yellow) and recruit SMC-ScpAB condensin complexes (black arcs).

\section{Plasmidic ParABS systems}

Plasmids are short (5-300kb), closed-circular DNA molecules that confer advantages to the host cell, such as antibiotic resistance, virulence attributes or new metabolic traits (Ochman, Lawrence, \& Groisman, 2000). Plasmids can be transferred horizontally between cells in a colony to propagate beneficial qualities into the population (Ochman et al., 2000). High-copy number plasmids harbour hundreds to thousands of copies per cell, therefore random partition between daughter cells is enough to ensure their propagation (Reyes-Lamothe et al., 2014; Y. Wang, 2017). Low copy number plasmids (typically 2-3 per cell), instead, rely on partitioning systems to ensure faithful partitioning by regulating their spatial localization within the host cell. 


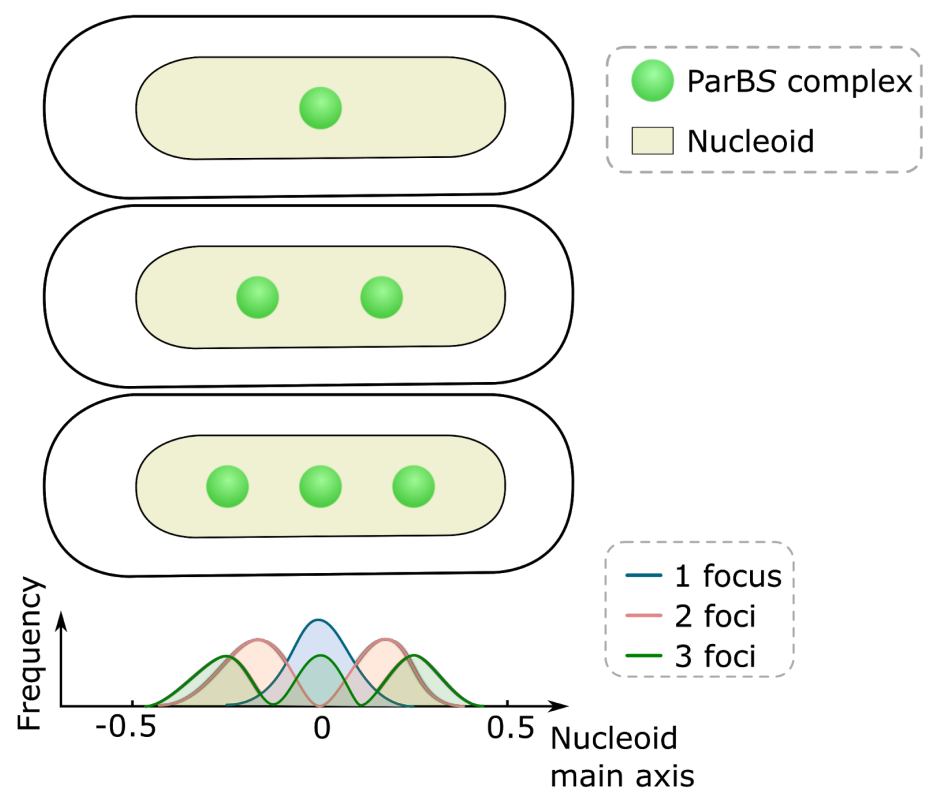

Figure 5. ParABS-mediated sub-cellular organization of low copy number plasmids. On average, plasmids are equidistributed along the main axis of the nucleoid, but the actual positions depend on the number of plasmids per cell. This spatial pattern ensures faithful partitioning during cell division of the host. Cell outline (black curve), nucleoid (grey), and partition complexes (green circles) are represented in the first three schemes. The bottom plot displays the average axial localization of plasmids for cells containing one, two or three partition complexes.

Escherichia coli plasmids F and P1 are two low-copy number plasmids for which the ParABS system is both essential and sufficient for stable inheritance (Austin \& Wierzbicki, 1983). The ParABS system is required for their specific sub-cellular localization, ensuring the presence of at least one copy in each cell half upon cell division. Shortly after plasmid replication, the ParABS system splits the single partition complex into two separate complexes (Ah-Seng et al., 2013; Fung, Bouet, \& Funnell, 2001; Onogi, Miki, \& Hiraga, 2002). The action of the ParABS system also ensures that, on average, plasmids are equidistributed along the main axis of the nucleoid (Figure 5) (Ringgaard, van Zon, Howard, \& Gerdes, 2009; Sengupta, Nielsen, Youngren, \& Austin, 2010). It is important to note that, after segregation, plasmids occupy specific sub-cellular locations, but that their movement remains highly dynamic. In fact, in rare cases segregated partition complexes can coalesce and fuse into a single partition complex (Sengupta et al., 2010). Importantly, the stable sub-cellular localization of partition complexes is severely affected in the absence of ParA (Le Gall et al., 2016). More generally, the deletion of any of the three components of the ParABS system 
leads to the loss of the plasmid, gradually at each cell division, due to aberrant partition complex positioning.

\subsection{ParA-mediated partitioning}

In eukaryotic DNA segregation, microtubules pull chromosomes towards the poles during mitosis (Heald \& Nogales, 2002). In this process, the growth of filaments regulated by GTP-hydrolysis promotes chromosome partitioning between the two daughter cells. The segregation of plasmids and chromosomes by the ParABS system also uses the energy of NTP hydrolysis. Early observations of ParA in vitro polymerization lead to models suggesting that DNA segregation by ParABS may also require the formation of long filaments (J.-Y. Bouet, Ah-Seng, Benmeradi, \& Lane, 2007; Ebersbach et al., 2006; G. E. Lim, Derman, \& Pogliano, 2005). More recent studies using super-resolution microscopy methods showed, however, that ParA does not form long filaments in living cells (Le Gall et al., 2016; H. C. Lim et al., 2014). Instead, a body of evidence now supports a chemophoresis or reaction-diffusion mechanism (see below) whereby the ParBS complex modulates the inhomogeneous and dynamic patterning of ParA (Vechiarelli, 2010, Sugawara and Kaneko, 2011, letswaart et al. 2014). This mechanism shares similarities with that used by PomXYZ and MinCDE to localize the division septum at mid-cell, but also differences in terms of the dynamics and sub-cellular distribution of positioning factors as well as in their mathematical descriptions (Bergeler and Frey 2018; Lutkenhaus 2007).

Critically, ParA-ATP binds non-specific DNA (nsDNA) efficiently, while ParA-ADP displays a considerable reduction in nsDNA binding (Davey \& Funnell, 1994). Interestingly, ParA's ATP hydrolysis rate is low in the absence of other factors, but is synergistically stimulated by the presence of nsDNA, parS and ParB (Davis, Martin, \& Austin, 1992). ParA-ADP recovers its nsDNA-binding capacity after ATP re-binding and a conformational change (Davey \& Funnell, 1997; Libante, Thion, \& Lane, 2001; Vecchiarelli et al., 2010). This latter transition is rather slow and introduces a delay in the ParA nsDNA binding cycle (Davey \& Funnell, 1997; Libante et al., 2001; Vecchiarelli et al., 2010). This delay allows the ParA-ADP form to be homogeneously re-distributed over the entire nucleoid. In particular, the region depleted of ParA in the vicinity of the ParBS complex is not immediately refilled and a ParA distribution gradient can persist (Hwang et al., 2013). In the presence of this 
ParA gradient, the tethering between ParA and ParBS is preferably established on the direction of the ParA gradient. This results in an asymmetric tethering that biases the diffusion of the partition complex and creates a directed movement (Hwang et al., 2013; Vecchiarelli, Neuman, \& Mizuuchi, 2014; Vecchiarelli, Seol, Neuman, \& Mizuuchi, 2014). These observations were originally used to propose a first version of a 'diffusion-ratchet' model where plasmid motion is driven by ParA gradients (Figure 6) (Vecchiarelli et al. 2010). Sugawara and Kaneko provided the first mathematical description of this model, and proposed that effective 'chemophoretic' forces exerted by ParA would be responsible for driving plasmid movement and plasmid equipartitioning (Sugawara and Kaneko 2011). More recently, letswaart et al. additionally showed that mid-cell and quarter-positioning of plasmids could occur due to the balancing of ParA fluxes on either side of the plasmid giving rise to the 'flux-balance' mechanism (letswaart et al. 2014). The same year, the Mizzuchi lab extended their diffusion-ratchet model by introducing a chemophoresis force to direct cargo motion towards regions of higher ParA concentrations (Vecchiarelli et al. 2014). Finally, Walter et al. coupled simple linear reaction-diffusion equations with a proteophoresis force (i.e. a volumetric chemophoresis) to describe partition complex translocation and positioning (Walter et al. 2018). Importantly, numerical simulations from all these different laboratories could faithfully reproduce the in vivo observation that plasmids move and reach stable sub-cellular positions without the requirement of ParA polymers.

Experimental support for these mechanisms arose, to a large extent, from elegant in vitro fluorescence microscopy experiments showing that ParBS complexes are able to move on a two-dimensional surface decorated by ParA (Vecchiarelli et al., 2013; Vecchiarelli, Neuman, et al., 2014). The topology of these experiments lead, understandably, to models where partitioning occurred on the two-dimensional surface of the nucleoid. But, it was not until it became possible to localize ParA and the partition complex within the nucleoid volume with high spatial resolution, that it was realized that plasmid partitioning rather occurs within the interior of the nucleoid (Le Gall et al., 2016). We note that this localization pattern of ParA and the partition complex do not affect the main features of the proposed models.

Recently, other studies on the chromosomal ParABS system of $C$. crescentus argued for a distinct mechanism. Lim et al. (2014) performed computer simulations where they 
were unable to detect, in their chromosomal ParABS model, in silico evidence for directed motion as predicted by the diffusion-reaction model. Thus, they proposed that chromosomal elasticity may represent a key actor in DNA segregation. The "DNA-relay model" posits that ParA-ATP binds to a DNA locus fluctuating in space (Figure 6). These fluctuations help distant DNA-bound ParA to bind to the partition complex. At the beginning of this binding, the DNA-ParA spring is out-of-equilibrium and, under the effect of the spring's restoring force, pulls on the partition complex. Like for reaction-diffusion models, the asymmetric distribution of ParA creates a preferential direction in which more ParA-ParB interactions are formed. Critically, C. crescentus ParA displays a distribution gradient that points in the direction of the main axis of the cell (H. C. Lim et al., 2014), with the lowest ParA density observed at the location of unsegregated origins. By combining computer simulations with the pre-existing biochemical properties of $C$. crescentus ParA and ParB, Lim et al. showed that the addition of DNA elasticity to their model recapitulated both the spontaneous formation of propagating ParA gradients, and their oscillation with the partition complex (Surovtsev, Campos, \& Jacobs-Wagner, 2016; Surovtsev, Lim, \& Jacobs-Wagner, 2016). While elegant, it is not clear how universal this mechanism may be, as several ParABS systems (e.g. P1 plasmid, B. subtilis) fail to exhibit partition complex or ParA gradient oscillations in vivo (Hatano \& Niki, 2010).

A similar concept was later introduced by the Mizuuchi lab to extend their previous work with a molecular explanation of plasmid motion. In the now termed 'Brownian-ratchet' model, the ParB cargo is tethered to DNA-bound ParA through elastic bonds (Hu, Vecchiarelli, Mizuuchi, Neuman, \& Liu, 2015, 2017a). This new ingredient allowed the authors to faithfully recapitulate and explain all the different motility behaviors observed experimentally for the plasmid, notably by varying ParA-ATP-ParB bond dissociation rate and the replenishment rate of the ParA- depletion zone.

A third model ("hitch-hiking model", Figure 6) was proposed from the distribution of ParABS within the volume of the nucleoid. While large macromolecular complexes -such as the RNA polymerase- are generally excluded from the nucleoid (Stracy et al., 2015), the different components of the ParABS system reside within the nucleoid for the entirety of the cell cycle (Le Gall et al., 2016). Critically, this localization pattern requires the energy of ATP hydrolysis, since both the ability of ParA to hydrolyze ATP and the stimulation of ParA's ATP activity by ParB are required to maintain the partition complex confined within the 
volume of the nucleoid (Le Gall et al., 2016). DNA within the nucleoid is not homogeneously distributed, but instead displays regions of low and high density (HDR - for High-Density chromosomal DNA Regions) (Le Gall et al., 2016; Marbouty et al., 2015).

Interestingly, single ParA particles display static and dynamic trajectories within this inhomogeneous nucleoid, and the former population preferentially localizes to HDRs (Le Gall et al., 2016). These observations were rationalized in terms of the ability of ParA-ATP to bind nsDNA (representing static trajectories), and of Par-ADP from losing this ability (thereby representing dynamic trajectories). Thus, ParA-ATP remains bound to regions with more chromosomal DNA (HDRs), and hydrolysis of ATP leads to a conversion to a form that can diffuse freely on the nucleoid and bind (after a slow conformational change) to other HDRs. This conversion would be favoured by the stimulation of ATP hydrolysis by ParBS. The "hitch-hiking model" is based on a diffusion-ratchet mechanism, but incorporates the non-homogeneous, volumetric distribution of the components of the partition system within the inhomogeneous nucleoid (Figure 6). Mathematical validation for this model is still awaiting.

A

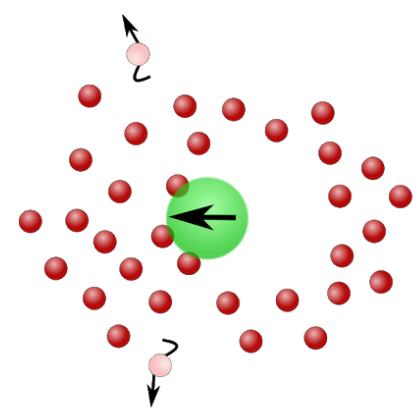

Diffusion-ratchet model
B

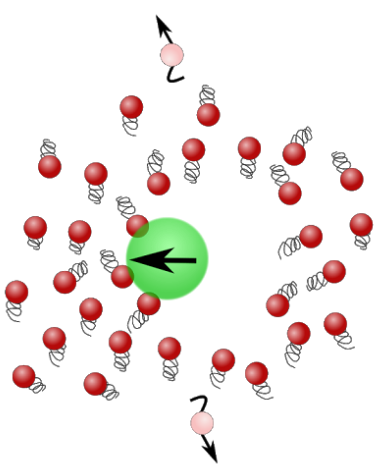

DNA-relay model
C

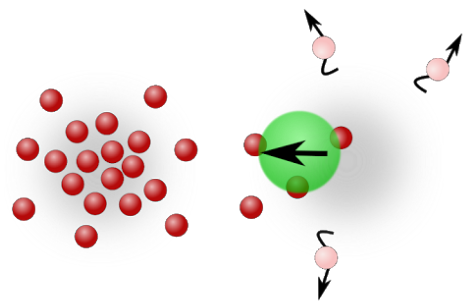

Hitch-hikking model

Figure 6. Models of ParA-mediated segregation. (A) In the 'diffusion-ratchet' model, directed movement of the partition complex (green sphere) results from a thermodynamic force generated by the asymmetric DNA-bound ParA distribution (red spheres) present near the complex. (B) In the 'DNA-relay' model, the partition complex exploits the energy of thermal fluctuations of the DNA-bound ParA (red spheres). (C) The hitch-hiking model proposes that HDRs (high-density chromosomal DNA regions, shade) create pools of DNA-bound ParA that direct the movement of partition complexes. 


\subsection{Partition complex assembly}

The formation of the partition complex on the centromeric sequence allows both the recognition of DNA molecules and the partitioning mechanism to proceed by promoting ParB-ParA interactions. The partition complex assembles by the binding of ParB to parS and the self-organization of multiple ParB dimers into higher-order structures. ParB contains three functional domains (J.-Y. Bouet \& Funnell, 2019; Funnell, 2016): a C-terminal dimerization domain (Fisher et al., 2017; Leonard, Butler, \& Löwe, 2004), a central helix-turn-helix domain that specifically binds parS (Schumacher \& Funnell, 2005), and a flexible N-terminal domain that interacts with ParA (Chen, Lin, Chu, Hsu, \& Sun, 2015; Song, Rodrigues, Graham, \& Loparo, 2017; Zhang \& Schumacher, 2017). The distribution of ParB binding around pars was revealed by chromatin immunoprecipitation sequencing (ChIP-seq), a next-generation sequencing method that determines the binding profile of proteins to genomic DNA (Furey, 2012). Surprisingly, ParB enrichment was observed not only at parS but also extended several kilobases away from the parS locus (Figure 7) (Breier \& Grossman, 2007; Lagage, Boccard, \& Vallet-Gely, 2016; Lynch \& Wang, 1995; Murray, Ferreira, \& Errington, 2006; Rodionov, Lobocka, \& Yarmolinsky, 1999). This extended binding of ParB is independent of ParA (Breier \& Grossman, 2007), and depends exclusively on the position of the parS sequence since ectopic parS sites generate similar DNA binding profiles (Debaugny et al., 2018). The existence of this extended ParB binding profile was observed for all chromosomal and plasmidic partition complexes investigated (Debaugny et al., 2018). Thus, the extended binding pattern of ParB around parS may be a universal property of ParABS systems. Importantly, ParB mutants defective in extended binding are also unable to assemble partition complexes (Breier \& Grossman, 2007). 


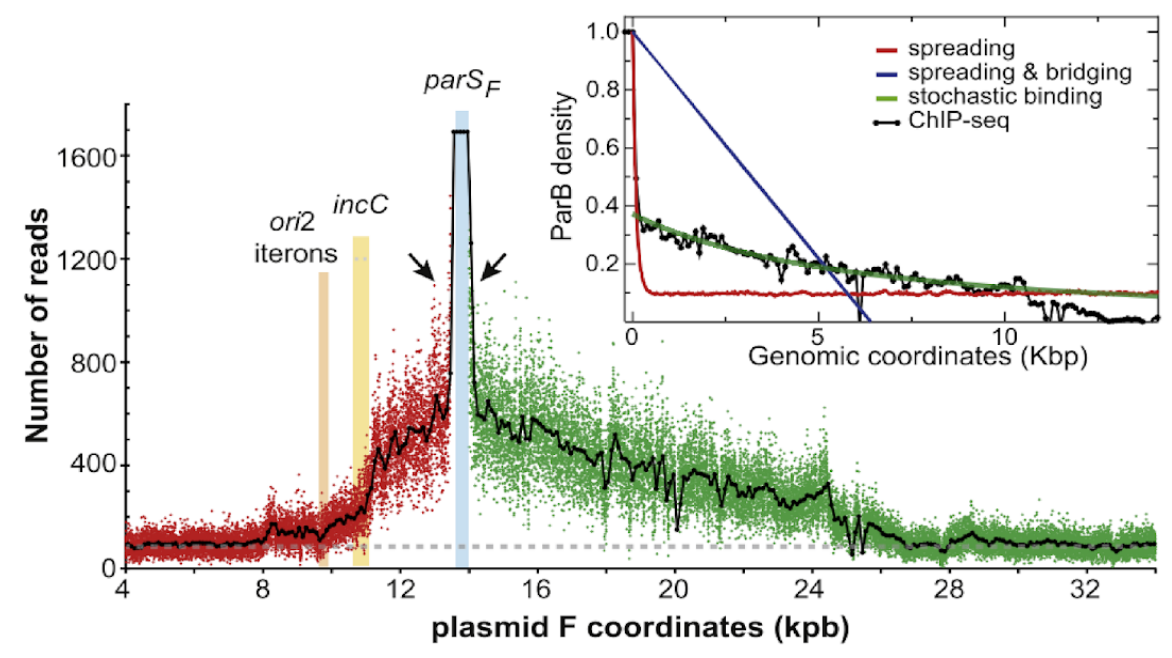

Figure 7. ChIP-Seq DNA-binding profile of ParB (Sanchez et al., 2015). x-axis represents

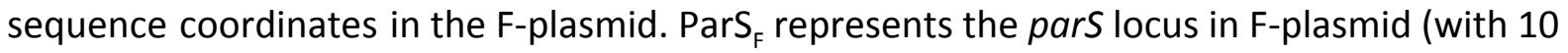
copies of parS). ori2 represents the origin of replication of the F-plasmid. Inset displays the Chip-seq binding experimental profile (black), and the binding profiles predicted by a pure 'spreading' model (red), the 'spreading and bridging' model (blue), and the 'nucleation and caging' model (green).

From this DNA binding profile, a first model of the partition complex structure was proposed in which ParB dimers formed a long filament that originates from parS sites (Murray et al., 2006; Rodionov et al., 1999). This "spreading" model was motivated by roadblock experiments that showed that the presence of a strong binding site inserted near the pars locus hindered the binding of ParB beyond the roadblock. However, given the number of ParB copies ( 300 ParB dimers per partition complex (Adachi, Hori, \& Hiraga, 2006; J. Y. Bouet, Rech, Egloff, \& Biek, 2005)), a strict linear arrangement along the DNA fibre could not generate a continuous and contiguous spreading over large genomic distances ( 10kb).

Recently, single-molecule flow stretching experiments showed that the nucleation of distinct nsDNA-bound ParB complexes leads to DNA condensation (Graham et al., 2014). This and other evidence lead to a second family of models proposing that formation of partition complexes involves both spreading and also long-range, bridging interactions between distant DNA segments that contact each other by 3D looping interactions ("spreading and bridging" model) (Broedersz et al., 2014; Debaugny et al., 2018; Song et al., 2017). Thus, this model posits that both nearest-neighbours and bridging interactions are 
required for the assembly of the partition complex. Using a coarse-grained modeling approach, Broedersz et al. proposed that these interactions are sufficient to assemble a complex composed of multiple small nucleoprotein filaments that interact and cluster together in 3D space (Broedersz et al., 2014; Debaugny et al., 2018; Song et al., 2017). Critically, other combinations of interactions ("adjacent", "bridging", "adjacent or bridging") failed to robustly assemble partition complexes.

The "spreading and bridging" model accounts for many of the key properties of the ParBS complex, but is, however, unable to qualitatively reproduce the ParB DNA binding profile (Sanchez et al., 2015). In fact, this profile exhibits an exponential decay from parS. Critically, models with only bridging or spreading interactions fail to fit this exponential decay, while the "bridging and spreading" model predicts a linear decay (Sanchez et al., 2015). In addition, evidence from footprinting analysis of ParB binding showed that parS sites, but not the neighboring DNA, are protected from proteolysis, suggesting that spreading interactions are not stable (Sanchez et al., 2015). Sanchez et al. proposed a new model ("Nucleation and Caging") that is able to reproduce the extent of ParB binding away from parS, as well as its exponential decay with genomic distance (Sanchez et al., 2015). In this model, ParB dimers are localized to the 3D volume surrounding parS sequences by: specific interactions with parS, weak interactions between ParB dimers, and non-specific interactions with the surrounding DNA. Within this spherical cage, the frequency of interaction of ParB with distant DNA segments is defined by the polymer physics of DNA (de Gennes \& Gennes, 1979; Schiessel, 2013). In fact, super-resolution microscopy showed that partition complexes imprison the large majority of ParB dimers (>90\%) in the cell (Sanchez et al., 2015). More recently, Walter et al. showed that reductions in the value of spreading interactions in the "spreading and bridging model" reproduce the experimental ParB DNA binding profile more faithfully, and fit experimental profiles as well as the "Nucleation and Caging model" when spreading interactions become negligible (Walter et al., 2018). Thus, modeling suggests that long-range 3D looping interactions between ParB dimers -but not spreading interaction- are required for the assembly of the partition complex.

Recently, molecular evidence for ParB-ParB dimer interactions and their roles in DNA condensation and assembly of partition complexes has been unearthed. A co-crystal structure of Helicobacter pylori ParB with parS (Chen et al., 2015; Song et al., 2017) reveals that ParB tetramerization is mediated by in cis and in trans interactions involving the 
$\mathrm{N}$-terminal domain. More recent studies tested the effect of systematic mutations of $\mathrm{N}$-terminal domain residues located in a ParB-ParB interaction hub on DNA compaction (Song et al., 2017) and partition complex assembly (Song et al., 2017)(Chen et al., 2015). These studies indicated that mutants in the ParB-ParB interaction hub exhibit abnormal DNA condensation (Song et al., 2017) and fail to assemble partition complexes (Debaugny et al., 2018).

A
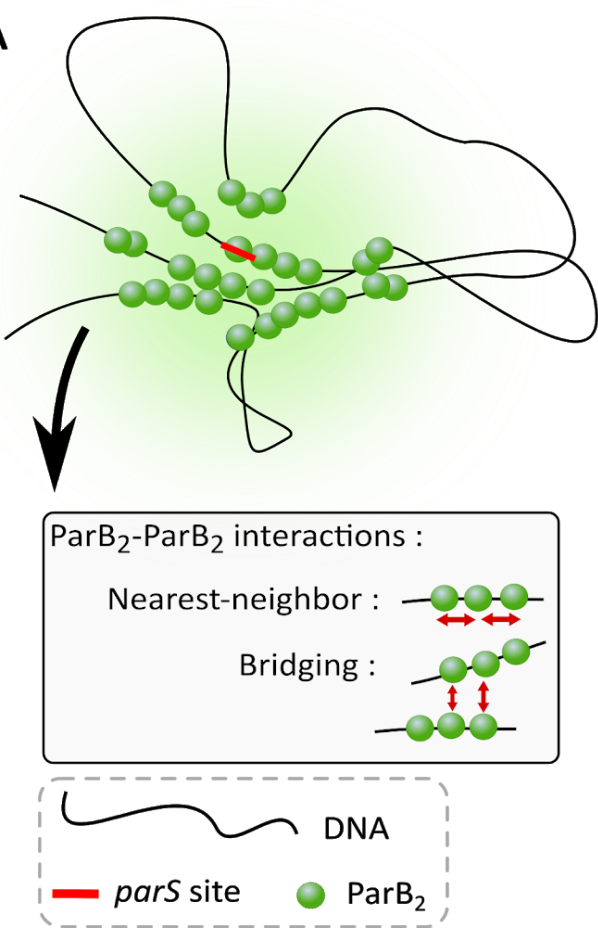

B
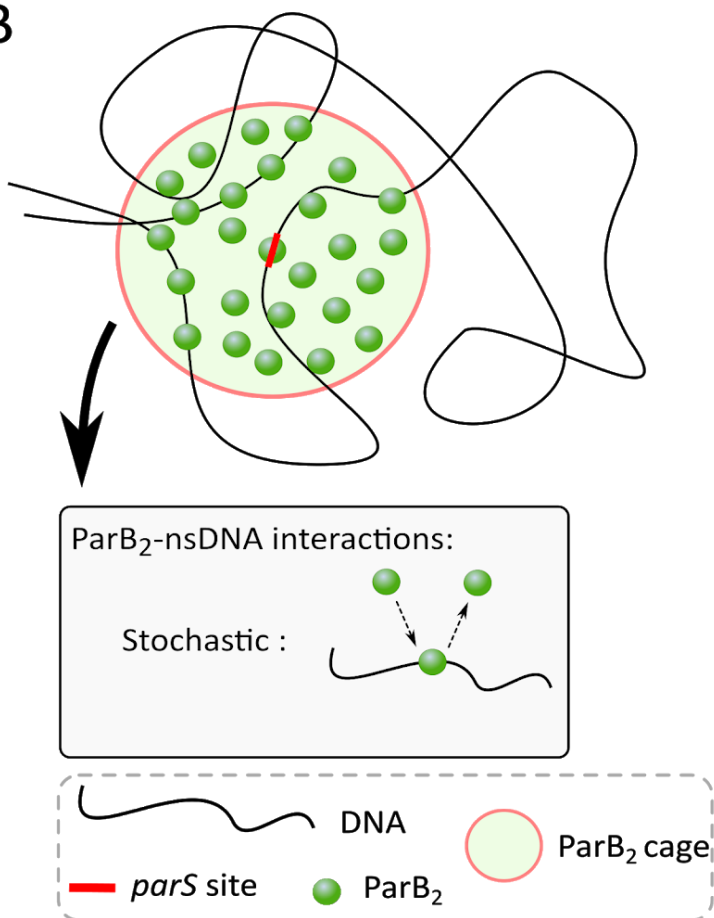

Figure 8. (A) Schematic view of the spreading and bridging model, in which the ParBS complex is formed by a combination of nearest-neighbor and bridging interactions. (B) Schematic view of the Nucleation and Caging model, in which ParBs reside in a defined spherical region and interact stochastically with a fluctuating DNA polymer. 


\subsection{Conclusion}

The ParABS system is a relatively simple apparatus -composed of only three essential components- in contrast to other bacterial machinery with tens of parts (e.g flagellar motor). Yet, despite this apparent simplicity, this system reveals an outstanding degree of complexity. Recent biophysical measurements and physical models helped us to start understanding how partition complexes assemble, and how these complexes are robustly segregated and localized in the sub-cellular space. However, we still don't fully understand the origin of oscillation waves or their purpose (i.e. how they contribute to segregation/positioning), the molecular mechanisms responsible for segregation of newly replicated parS-harbouring DNA molecules, or for coupling segregation with chromosomal arm cohesion. In future, experimental and theoretical physical approaches will surely continue to provide important insights into these important questions. Importantly, they will also continue to provide a model for how these approaches can be used to study other, more complex, molecular mechanisms.

\section{References}

Adachi, S., Hori, K., \& Hiraga, S. (2006). Subcellular positioning of F plasmid mediated by dynamic localization of SopA and SopB. Journal of Molecular Biology, 356(4), 850-863.

Ah-Seng, Y., Rech, J., Lane, D., \& Bouet, J.-Y. (2013). Defining the role of ATP hydrolysis in mitotic segregation of bacterial plasmids. PLoS Genetics, 9(12), e1003956.

Austin, S., \& Wierzbicki, A. (1983). Two mini-F-encoded proteins are essential for equipartition. Plasmid, 10(1), 73-81.

Badrinarayanan, A., Le, T. B. K., \& Laub, M. T. (2015). Bacterial chromosome organization and segregation. Annual Review of Cell and Developmental Biology, 31, 171-199.

Baxter, J. C., \& Funnell, B. E. (2014). Plasmid Partition Mechanisms. Microbiology Spectrum, 2(6). https://doi.org/10.1128/microbiolspec.PLAS-0023-2014

Ben-Yehuda, S., Rudner, D. Z., \& Losick, R. (2003). RacA, a bacterial protein that anchors chromosomes to the cell poles. Science, 299(5606), 532-536.

Bouet, J.-Y., Ah-Seng, Y., Benmeradi, N., \& Lane, D. (2007). Polymerization of SopA partition ATPase: regulation by DNA binding and SopB. Molecular Microbiology, 63(2), 468-481.

Bouet, J.-Y., \& Funnell, B. E. (2019). Plasmid Localization and Partition in Enterobacteriaceae. EcoSal Plus, 8(2). https://doi.org/10.1128/ecosalplus.ESP-0003-2019

Bouet, J. Y., Rech, J., Egloff, S., \& Biek, D. P. (2005). Probing plasmid partition with centromere-based incompatibility. Molecular. Retrieved from https://onlinelibrary.wiley.com/doi/abs/10.1111/j.1365-2958.2004.04396.x

Breier, A. M., \& Grossman, A. D. (2007). Whole-genome analysis of the chromosome partitioning and sporulation protein SpoOJ (ParB) reveals spreading and origin-distal sites on the Bacillus subtilis chromosome. Molecular Microbiology, 64(3), 703-718.

Broedersz, C. P., Wang, X., Meir, Y., Loparo, J. J., Rudner, D. Z., \& Wingreen, N. S. (2014). Condensation and localization of the partitioning protein ParB on the bacterial chromosome. Proceedings of the National 
Academy of Sciences of the United States of America, 111(24), 8809-8814.

Brooks, A. C., \& Hwang, L. C. (2017). Reconstitutions of plasmid partition systems and their mechanisms. Plasmid, 91, 37-41.

Chen, B.-W., Lin, M.-H., Chu, C.-H., Hsu, C.-E., \& Sun, Y.-J. (2015). Insights into ParB spreading from the complex structure of SpoOJ and parS. Proceedings of the National Academy of Sciences of the United States of America, 112(21), 6613-6618.

Davey, M. J., \& Funnell, B. E. (1994). The P1 plasmid partition protein ParA. A role for ATP in site-specific DNA binding. The Journal of Biological Chemistry, 269(47), 29908-29913.

Davey, M. J., \& Funnell, B. E. (1997). Modulation of the P1 plasmid partition protein ParA by ATP, ADP, and P1 ParB. The Journal of Biological Chemistry, 272(24), 15286-15292.

Davis, M. A., Martin, K. A., \& Austin, S. J. (1992). Biochemical activities of the parA partition protein of the P1 plasmid. Molecular Microbiology, 6(9), 1141-1147.

Debaugny, R. E., Sanchez, A., Rech, J., Labourdette, D., Dorignac, J., Geniet, F., ... Bouet, J.-Y. (2018). A conserved mechanism drives partition complex assembly on bacterial chromosomes and plasmids. Molecular Systems Biology, 14(11), e8516.

Ebersbach, G., \& Gerdes, K. (2005). Plasmid segregation mechanisms. Annual Review of Genetics, 39, 453-479.

Ebersbach, G., Ringgaard, S., Moller-Jensen, J., Wang, Q., Sherratt, D. J., \& Gerdes, K. (2006). Regular cellular distribution of plasmids by oscillating and filament-forming ParA ATPase of plasmid pB171. Molecular Microbiology, Vol. 61, pp. 1428-1442. https://doi.org/10.1111/j.1365-2958.2006.05322.x

Fisher, G. L., Pastrana, C. L., Higman, V. A., Koh, A., Taylor, J. A., Butterer, A., ... Dillingham, M. S. (2017). The structural basis for dynamic DNA binding and bridging interactions which condense the bacterial centromere. eLife, 6. https://doi.org/10.7554/eLife.28086

Fung, E., Bouet, J. Y., \& Funnell, B. E. (2001). Probing the ATP-binding site of P1 ParA: partition and repression have different requirements for ATP binding and hydrolysis. The EMBO Journal, 20(17), 4901-4911.

Funnell, B. E. (2016). ParB Partition Proteins: Complex Formation and Spreading at Bacterial and Plasmid Centromeres. Frontiers in Molecular Biosciences, 3, 44.

Furey, T. S. (2012). ChIP-seq and beyond: new and improved methodologies to detect and characterize protein-DNA interactions. Nature Reviews. Genetics, 13(12), 840-852.

Gerdes, K., Møller-Jensen, J., \& Bugge Jensen, R. (2000). Plasmid and chromosome partitioning: surprises from phylogeny. Molecular Microbiology, 37(3), 455-466.

Graham, T. G. W., Wang, X., Song, D., Etson, C. M., van Oijen, A. M., Rudner, D. Z., \& Loparo, J. J. (2014). ParB spreading requires DNA bridging. Genes \& Development, 28(11), 1228-1238.

Gruber, S. (2018). SMC complexes sweeping through the chromosome: going with the flow and against the tide. Current Opinion in Microbiology, 42, 96-103.

Gruber, S., \& Errington, J. (2009). Recruitment of Condensin to Replication Origin Regions by ParB/SpoOJ Promotes Chromosome Segregation in B. subtilis. Cell, Vol. 137, pp. 685-696. https://doi.org/10.1016/j.cell.2009.02.035

Gruber, S., Veening, J.-W., Bach, J., Blettinger, M., Bramkamp, M., \& Errington, J. (2014). Interlinked sister chromosomes arise in the absence of condensin during fast replication in B. subtilis. Current Biology: $C B$, 24(3), 293-298.

Hatano, T., \& Niki, H. (2010). Partitioning of P1 plasmids by gradual distribution of the ATPase ParA. Molecular Microbiology, 78(5), 1182-1198.

Heald, R., \& Nogales, E. (2002). Microtubule dynamics. Journal of Cell Science, 115(Pt 1), 3-4.

Hu, L., Vecchiarelli, A. G., Mizuuchi, K., Neuman, K. C., \& Liu, J. (2015). Directed and persistent movement arises from mechanochemistry of the ParA/ParB system. Proceedings of the National Academy of Sciences of the United States of America, 112(51), E7055-E7064.

Hu, L., Vecchiarelli, A. G., Mizuuchi, K., Neuman, K. C., \& Liu, J. (2017a). Brownian Ratchet Mechanism for Faithful Segregation of Low-Copy-Number Plasmids. Biophysical Journal, 112(7), 1489-1502.

Hu, L., Vecchiarelli, A. G., Mizuuchi, K., Neuman, K. C., \& Liu, J. (2017b). Brownian ratchet mechanisms of ParA-mediated partitioning. Plasmid, 92, 12-16.

Hwang, L. C., Vecchiarelli, A. G., Han, Y., Mizuuchi, M., Harada, Y., Funnell, B. E., \& Mizuuchi, K. (2013). ParA-mediated plasmid partition driven by protein pattern self-organization. The EMBO Journal, 32(9), 1238-1249.

Lagage, V., Boccard, F., \& Vallet-Gely, I. (2016). Regional Control of Chromosome Segregation in Pseudomonas aeruginosa. PLoS Genetics, 12(11), e1006428.

Lee, P. S., Lin, D. C. H., Moriya, S., \& Grossman, A. D. (2003). Effects of the chromosome partitioning protein SpoOJ (ParB) on oriC positioning and replication initiation in Bacillus subtilis. Journal of Bacteriology, 
185(4), 1326-1337.

Le Gall, A., Cattoni, D. I., Guilhas, B., Mathieu-Demazière, C., Oudjedi, L., Fiche, J.-B., ... Nollmann, M. (2016). Bacterial partition complexes segregate within the volume of the nucleoid. Nature Communications, 7, 12107.

Leonard, T. A., Butler, P. J. G., \& Löwe, J. (2004). Structural analysis of the chromosome segregation protein Spo0J from Thermus thermophilus. Molecular Microbiology, 53(2), 419-432.

Libante, V., Thion, L., \& Lane, D. (2001). Role of the ATP-binding site of SopA protein in partition of the F plasmid. Journal of Molecular Biology, 314(3), 387-399.

Lim, G. E., Derman, A. I., \& Pogliano, J. (2005). Bacterial DNA segregation by dynamic SopA polymers. Proceedings of the National Academy of Sciences of the United States of America, 102(49), 17658-17663.

Lim, H. C., Surovtsev, I. V., Beltran, B. G., Huang, F., Bewersdorf, J., \& Jacobs-Wagner, C. (2014). Evidence for a DNA-relay mechanism in ParABS-mediated chromosome segregation. elife, 3, e02758.

Lin, D. C., Levin, P. A., \& Grossman, A. D. (1997). Bipolar localization of a chromosome partition protein in Bacillus subtilis. Proceedings of the National Academy of Sciences of the United States of America, 94(9), 4721-4726.

Livny, J., Yamaichi, Y., \& Waldor, M. K. (2007). Distribution of centromere-like parS sites in bacteria: insights from comparative genomics. Journal of Bacteriology, 189(23), 8693-8703.

Lynch, A. S., \& Wang, J. C. (1995). SopB protein-mediated silencing of genes linked to the sopC locus of Escherichia coli F plasmid. Proceedings of the National Academy of Sciences of the United States of America, 92(6), 1896-1900.

Marbouty, M., Le Gall, A., Cattoni, D. I., Cournac, A., Koh, A., Fiche, J.-B., ... Nollmann, M. (2015). Condensinand Replication-Mediated Bacterial Chromosome Folding and Origin Condensation Revealed by $\mathrm{Hi}-\mathrm{C}$ and Super-resolution Imaging. Molecular Cell, Vol. 59, pp. 588-602. https://doi.org/10.1016/j.molcel.2015.07.020

Miermans, C. A., \& Broedersz, C. P. (2018). Bacterial chromosome organization by collective dynamics of SMC condensins. Journal of the Royal Society, Interface / the Royal Society, 15(147). https://doi.org/10.1098/rsif.2018.0495

Mohl, D. A., \& Gober, J. W. (1997). Cell Cycle-Dependent Polar Localization of Chromosome Partitioning Proteins in Caulobacter crescentus. Cell, 88(5), 675-684.

Murray, H., Ferreira, H., \& Errington, J. (2006). The bacterial chromosome segregation protein Spo0J spreads along DNA from parS nucleation sites. Molecular Microbiology, 61(5), 1352-1361.

Ochman, H., Lawrence, J. G., \& Groisman, E. A. (2000). Lateral gene transfer and the nature of bacterial innovation. Nature, 405(6784), 299-304.

Onogi, T., Miki, T., \& Hiraga, S. (2002). Behavior of sister copies of mini-F plasmid after synchronized plasmid replication in Escherichia coli cells. Journal of Bacteriology, 184(11), 3142-3145.

Reyes-Lamothe, R., Tran, T., Meas, D., Lee, L., Li, A. M., Sherratt, D. J., \& Tolmasky, M. E. (2014). High-copy bacterial plasmids diffuse in the nucleoid-free space, replicate stochastically and are randomly partitioned at cell division. Nucleic Acids Research, 42(2), 1042-1051.

Ringgaard, S., van Zon, J., Howard, M., \& Gerdes, K. (2009). Movement and equipositioning of plasmids by ParA filament disassembly. Proceedings of the National Academy of Sciences of the United States of America, 106(46), 19369-19374.

Rodionov, O., Lobocka, M., \& Yarmolinsky, M. (1999). Silencing of genes flanking the P1 plasmid centromere. Science, 283(5401), 546-549.

Sanchez, A., Cattoni, D. I., Walter, J.-C., Rech, J., Parmeggiani, A., Nollmann, M., \& Bouet, J.-Y. (2015). Stochastic Self-Assembly of ParB Proteins Builds the Bacterial DNA Segregation Apparatus. Cell Systems, 1(2), 163-173.

Schumacher, M. A., \& Funnell, B. E. (2005). Structures of ParB bound to DNA reveal mechanism of partition complex formation. Nature, 438(7067), 516-519.

Sengupta, M., Nielsen, H. J., Youngren, B., \& Austin, S. (2010). P1 plasmid segregation: accurate redistribution by dynamic plasmid pairing and separation. Journal of Bacteriology, 192(5), 1175-1183.

Song, D., Rodrigues, K., Graham, T. G. W., \& Loparo, J. J. (2017). A network of cis and trans interactions is required for ParB spreading. Nucleic Acids Research, 45(12), 7106-7117.

Stracy, M., Lesterlin, C., Garza de Leon, F., Uphoff, S., Zawadzki, P., \& Kapanidis, A. N. (2015). Live-cell superresolution microscopy reveals the organization of RNA polymerase in the bacterial nucleoid. Proceedings of the National Academy of Sciences of the United States of America, 112(32), E4390-E4399.

Sullivan, N. L., Marquis, K. a., \& Rudner, D. Z. (2009). Recruitment of SMC by ParB-parS organizes the origin region and promotes efficient chromosome segregation. Cell, 137(4), 697-707. 
Surovtsev, I. V., Campos, M., \& Jacobs-Wagner, C. (2016). DNA-relay mechanism is sufficient to explain ParA-dependent intracellular transport and patterning of single and multiple cargos. Proceedings of the National Academy of Sciences of the United States of America, 113(46), E7268-E7276.

Surovtsev, I. V., Lim, H. C., \& Jacobs-Wagner, C. (2016). The Slow Mobility of the ParA Partitioning Protein Underlies Its Steady-State Patterning in Caulobacter. Biophysical Journal, 110(12), 2790-2799.

Toro, E., Hong, S.-H., McAdams, H. H., \& Shapiro, L. (2008). Caulobacter requires a dedicated mechanism to initiate chromosome segregation. Proceedings of the National Academy of Sciences of the United States of America, 105(40), 15435-15440.

Vecchiarelli, A. G., Han, Y.-W., Tan, X., Mizuuchi, M., Ghirlando, R., Biertümpfel, C., ... Mizuuchi, K. (2010). ATP control of dynamic P1 ParA-DNA interactions: a key role for the nucleoid in plasmid partition. Molecular Microbiology, p. no - no. https://doi.org/10.1111/j.1365-2958.2010.07314.x

Vecchiarelli, A. G., Hwang, L. C., \& Mizuuchi, K. (2013). Cell-free study of F plasmid partition provides evidence for cargo transport by a diffusion-ratchet mechanism. Proceedings of the National Academy of Sciences of the United States of America, 110(15), E1390-E1397.

Vecchiarelli, A. G., Neuman, K. C., \& Mizuuchi, K. (2014). A propagating ATPase gradient drives transport of surface-confined cellular cargo. Proceedings of the National Academy of Sciences of the United States of America, 111(13), 4880-4885.

Vecchiarelli, A. G., Seol, Y., Neuman, K. C., \& Mizuuchi, K. (2014). A moving ParA gradient on the nucleoid directs subcellular cargo transport via a chemophoresis force. Bioarchitecture, 4(4-5), 154-159.

Walter, J.-C., Walliser, N.-O., David, G., Dorignac, J., Geniet, F., Palmeri, J., ... Broedersz, C. P. (2018). Looping and clustering model for the organization of protein-DNA complexes on the bacterial genome. New Journal of Physics, Vol. 20, p. 035002. https://doi.org/10.1088/1367-2630/aaad39

Wang, X., Brandão, H. B., Le, T. B. K., Laub, M. T., \& Rudner, D. Z. (2017). Bacillus subtilis SMC complexes juxtapose chromosome arms as they travel from origin to terminus. Science, 355(6324), 524-527.

Wang, X., Le, T. B. K., Lajoie, B. R., Dekker, J., Laub, M. T., \& Rudner, D. Z. (2015). Condensin promotes the juxtaposition of DNA flanking its loading site in Bacillus subtilis. Genes \& Development, 29(15), 1661-1675.

Wang, X., Montero Llopis, P., \& Rudner, D. Z. (2014). Bacillus subtilis chromosome organization oscillates between two distinct patterns. Proceedings of the National Academy of Sciences of the United States of America, 111(35), 12877-12882.

Wang, X., \& Rudner, D. Z. (2014). Spatial organization of bacterial chromosomes. Current Opinion in Microbiology, 22, 66-72.

Wang, X., Tang, O. W., Riley, E. P., \& Rudner, D. Z. (2014). The SMC condensin complex is required for origin segregation in Bacillus subtilis. Current Biology: CB, 24(3), 287-292.

Wang, Y. (2017). Spatial distribution of high copy number plasmids in bacteria. Plasmid, 91, 2-8.

Webb, C. D., Teleman, A., Gordon, S., Straight, A., Belmont, A., Lin, D. C., ... Losick, R. (1997). Bipolar localization of the replication origin regions of chromosomes in vegetative and sporulating cells of $B$. subtilis. Cell, 88(5), 667-674.

Wingreen, N. S., \& Huang, K. C. (2015). Physics of Intracellular Organization in Bacteria. Annual Review of Microbiology, 69, 361-379.

Yamaichi, Y., Fogel, M. A., \& Waldor, M. K. (2007). par genes and the pathology of chromosome loss in Vibrio cholerae. Proceedings of the National Academy of Sciences of the United States of America, 104(2), 630-635.

Zhang, H., \& Schumacher, M. A. (2017). Structures of partition protein ParA with nonspecific DNA and ParB effector reveal molecular insights into principles governing Walker-box DNA segregation. Genes \& Development, 31(5), 481-492. 